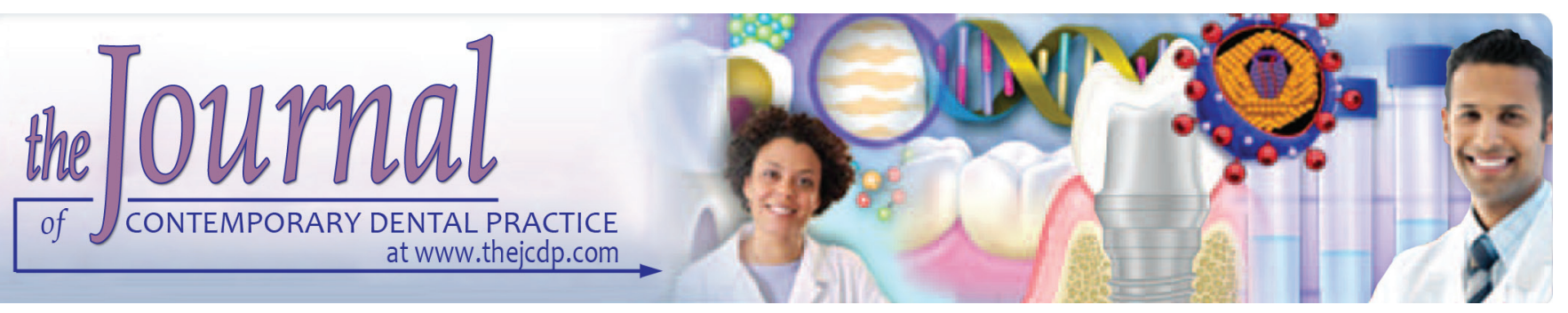

\title{
Incidence of Accidental Pulp Exposure During Tooth Preparation by Undergraduate Students for Fixed Partial Dentures
}

Aiman Othman Johar

\begin{abstract}
Aim: To identify the incidence of accidental pulp exposure during the preparation of vital abutment tooth for porcelainfused-to-metal (PFM) fixed prosthesis when prepared by undergraduate students.

Materials and methods: A cohort study was conducted on 471 teeth, which were prepared to received PFM fixed partial denture, in patients between the age group of 20-40 years for 72 months. All the teeth were vital and healthy periodontally; preoperative radiographs were recorded. Measurements were taken from cone beam computed tomography (CBCT) scans of human extracted teeth for reference of the pulpal depths. Teeth with endodontic pulp exposure were documented and referred to the endodontic department.
\end{abstract}

Results: In multivariate regression, when all variables were simultaneously considered and accounted for, tooth factors (arch and whether the tooth is anterior or posterior) and the distance between pulpal wall and cusp tip were significantly associated with odds of exposure $(p=0.006,0.03$ and $<0.0001$ ). Maxillary and anterior were associated with higher odds of lateral exposure compared to mandibular and posterior teeth $(O R=10.34$ and 3.53).

Conclusion: The present study draws the attention of the clinician towards the detrimental effect of tooth preparation on the vital pulp. The study concluded that maxillar and anterior teeth exposure has higher odds than mandibular and posterior teeth.

Clinical significance: The undergraduate students must be trained to be extra careful while preparation of anterior and maxillary teeth for fixed partial dentures (FPD) to avoid pulpal exposure.

Oral and Maxillofacial Prosthodontics Department, King Abdulaziz University, Jeddah, Kingdom of Saudi Arabia

Corresponding Author: Aiman Othman Johar, Oral and Maxillofacial Prosthodontics Department, King Abdulaziz University, Jeddah, Kingdom of Saudi Arabia, Phone: +966555665565, e-mail: Aojohar@kau.edu.sa
Keywords: Fixed partial dentures, Incidence, Pulp exposure, Tooth preparation.

How to cite this article: Johar AO. Incidence of Accidental Pulp Exposure During Tooth Preparation by Undergraduate Students for Fixed Partial Dentures. J Contemp Dent Pract 2019;20(2):258-262.

Source of support: São Paulo Research Foundation (FAPESP), grant number 2014/24478-0.

\section{Conflict of interest: None}

\section{INTRODUCTION}

Fixed partial dentures (FPD) have been the mainstay for there placement of missing teeth for many years due to their clinical biocompatibility, longevity, satisfactory mechanical properties, affordability, and reliability. ${ }^{1-5}$ One of the most common complications of fixed partial denture is the need for endodontic treatment, ${ }^{6-8}$ due to pulp insult either during or after the fixed partial denture treatment. ${ }^{6}$ A myriad of factors may compromise the vitality of the pulp during the construction of an FPD. These include the tooth anatomy, dentists' clinical skills, temperature changes, type of the cement, the instruments used, dehydration of the tooth, occlusal prematurities, and inaccurate tooth preparation..$^{6-9}$ Despite the attempts at a conservative and preservative technique, and there will always be threats to pulpal integrity duringfixed prosthesis construction. ${ }^{3-5}$

The knowledge of tooth anatomy is paramount in proper tooth preparation. Metal-ceramic restoration requires a standard average reduction of $1.5-2 \mathrm{~mm}$ of incisal/occlusal reduction, axial reduction of 1.2-1.5 $\mathrm{mm}$, and proximal reduction of $1.5 \mathrm{~mm}$ depends on the type of restoration. 10,11 The total thickness of the dental tissues differs according to the location and size of the tooth. Therefore, clinical examination and radiographic 
diagnosis are needed to determine the incidence of injuring pulp. ${ }^{12}$ Modern in vitro studies use digital radiographs to measure the morphological anatomic landmarks of pulp chambers. ${ }^{13-17}$ Clinicians use CBCT as well as periapical radiograph to determine these measurements. CBCT has been documented to overcome the overlapping and orientation errors encountered with conventional 2D radiographic methods. ${ }^{18}$

Many studies measured and discussed the complications of vital pulp impairment after teeth preparation for FPD or full crown either immediately after the treatment or through long-term follow-up. ${ }^{6-9,11,12,19}$ However, an exhaustive literature search revealed very few studies reported on immediate pulpal injury during tooth preparations. ${ }^{20}$ As most pulpal exposures occur during the preparation phase; the clinical skill and training of the clinicians play an important factor in the success of tooth preparation. Hence, the objective of this study was to assess the incidence of accidental pulp exposure during the preparation of vital abutment teeth by undergraduate students for PFM fixed partial denture among patients between 20 years and 40 years old.

\section{MATERIALS AND METHODS}

\section{Study Design and Study Population}

A cohort study was conducted to study the incidence of accidental pulp exposures occurring during the preparation of vital teeth for FPD among patients aged 20-40 years. This study was conducted on data obtained from all the patients, aged 20-40 years, treated for fixed partial denture (FPD) at the Prosthodontics Department, King Abdulaziz University Dental College, Jeddah, Saudi Arabia, from 2010 through 2015.

The sample size was calculated based on the previous study. $^{21}$

\section{Ethical Clearance and Informed Consent}

The ethical clearance was obtained from the Institutional Ethics Committee of King Abdulaziz University Dental College, Jeddah, Kingdom of Saudi Arabia. A legal written informed consent was obtained from all participants after explaining the study protocol in English and the local language.

\section{Inclusion and Exclusion Criteria}

Inclusion criteria involved all the abutment teeth evaluated as vital sound teeth and healthy periodontally. The patients who had vital abutment teeth received metalceramic FPDs or single crowns, and had not received any root canal treatment or filling prior to the FPD restoration were included in the study. Supraerupted, malaligned, malposed teeth, carious teeth, teeth with pre-existing restoration, non-vital teeth and teeth with clinical attachment loss or any periodontal problems were excluded from the study.

\section{Assessment of the Abutment Teeth}

Before tooth preparation, the preoperative periapical radiograph was recorded to assess the anterior abutment teeth and periapical and bitewing radiographs were used to evaluate the posterior abutment teeth. Measurements were calculated from the periapical radiograph of anterior teeth and bitewing radiographs of posteriors. Alginate impression of all the abutment teeth was made carefully. Reference Measurements

The CBCT was done on human extracted sound teeth that represent all the quadrants, and average measurements were taken and documented.

\section{Tooth Preparation}

Teeth were prepared using diamond burs (Brasseler, California, USA) in a high-speed handpiece under air and water coolant. Undergraduate dental students prepared the teeth to receive restoration. The students used the standard technique for tooth preparation. For posterior abutment preparation, occlusal reduction of $1.5 \mathrm{~mm}$ for metal and $2 \mathrm{~mm}$ for PFM was done using round-end tapered diamond bur. The proximal reduction was done using short needle diamond bur followed by the axial reduction of $1.5 \mathrm{~mm}$ using flat-end tapered diamond bur. For the anterior teeth, incisal reduction of 1.5-2 mm was done followed by proximal reduction with a long needle diamond bur and 1.2-1.5 mm axial reduction using flat-end taper diamond bur. The lingual reduction was performed using football-shaped diamond burlmm for PFM and $0.7 \mathrm{~mm}$ for metal using. Finish line prepared as a shoulder for all ceramic, and chamfer finish line for metal. Teeth were prepared using high-speed handpiece with air-water spray coolant.

If pulpal exposure occurred, patients were informed of the study, and thereafter obtaining consent, data on the student gender, patient's gender and age, arch, side, the tooth being anterior or posterior, the distance between pulpal wall and cusp tip as well as the distance between pulpal wall and lateral enamel were recorded. The patients were then referred for immediate treatment. All of the endodontic treatments were accomplished by the same clinician.

\section{Statistical Analysis}

The data were entered in Microsoft Office, Excel worksheets and analyzed using software IBM SPSS v. 20.0 (IBM Statistics, SPSS, Chicago, USA). The normality 
of the data was assessed using the Kolmogorov-Smirnov test while Levene's test for equality of error variances was used to analyze the homogeneity of error variances. Descriptive statistics were calculated as frequencies and percentages or means and standard deviations. Association between lateral and occlusal exposures was assessed using Chi-square. Differences in distances between pulpal wall and cusp tips as well as lateral walls in exposed and non-exposed cases were assessed using t-test. Univariate regression models were developed to investigate the association between outcome (lateral exposure) and independent variables (student gender, patient gender, patient age, arch, side, the tooth being anterior or posterior, the distance between pulpal wall and cusp tip as well as the distance between pulpal wall and lateral enamel). A multivariate regression model was developed including all the independent variables simultaneously. Odds ratios and confidence intervals were calculated.

\section{RESULTS}

The descriptive statistics are shown in Table 1. A total of 471 teeth from 320 patients were included in the study. Of the teeth included in the study, $5.1 \%$ of lateral and $0.2 \%$ occlusal exposures occurred. The study sample consisted of 471 teeth, which were prepared to receive a PFM fixed partial denture out of which $47.1 \%$ patients were males, and $52.9 \%$ were females, the average age of the patients was 35 years, out of 471 teeth 213 were maxillary teeth and 258 were mandibular teeth. Out of the total teeth that were prepared 214 were on the right side, and 257 were on the left side of the dental arch. The most commonly involved tooth was second premolar (144) followed by second molar (128), first premolar (59), first molar (54), canines (39), lateral incisor (25), central incisor (13) and third molar (9).

Table 2 shows the difference in distance from the pulpal wall to enamel. It was observed that in cases where exposure occurred, the distance from pulp to enamel was less than in cases where this did not occur.
The factors significantly associated with the occurrence of lateral exposure in the univariate regression are shown in Table 3. Increase in patient's age, the distance between pulpal wall and cusp tip and distance between pulpal wall and lateral enamel were associated with lower odds of lateral exposure $(\mathrm{OR}=0.84,0.10$ and 0.53). Higher odds of exposure were observed among male students $(\mathrm{OR}=2.86)$ and in anterior compared to posterior teeth $(\mathrm{OR}=7.05)$.

In multivariate regression, when all variables were simultaneously considered and accounted for, tooth factors (arch and whether the tooth is anterior or posterior) and the distance between pulpal wall and cusp

Table 1: Sample description $(n=471$ teeth)

\begin{tabular}{lll}
\hline \multicolumn{2}{c}{ Variables } & Statistics \\
\hline Student gender & Male: $\mathrm{n}(\%)$ & $222(47.1 \%)$ \\
& Female: $\mathrm{n}(\%)$ & $249(52.9 \%)$ \\
\hline Patient gender & Male: $\mathrm{n}(\%)$ & $218(46.3 \%)$ \\
& Female: $\mathrm{n}(\%)$ & $253(53.7 \%)$ \\
\hline Patient age & Range & $20(61 \%)$ \\
& Mean (SD) & $35.0(9.2)$ \\
\hline Arch & Maxillary: $\mathrm{n}(\%)$ & $213(45.2 \%)$ \\
& Mandibular: $\mathrm{n}(\%)$ & $258(54.8 \%)$ \\
\hline Side & Right: $\mathrm{n}(\%)$ & $214(45.4 \%)$ \\
& Left: $\mathrm{n}(\%)$ & $257(54.6 \%)$ \\
\hline Tooth & Central & $13(2.8 \%)$ \\
& Lateral & $25(5.3 \%)$ \\
& Canine & $39(8.3 \%)$ \\
& First premolar & $59(12.5 \%)$ \\
& Second premolar & $144(30.6 \%)$ \\
& First molar & $54(11.5 \%)$ \\
& Second molar & $128(27.2 \%)$ \\
& Third molar & $9(1.9 \%)$ \\
\hline
\end{tabular}

Table 2: Difference in distance from pulpal wall to enamel in cases where lateral exposure occurred and those where there was no exposure

\begin{tabular}{llll}
\hline & \multicolumn{2}{c}{ Distance in $\mathrm{mm}$} & $p$ value \\
\hline & No exposure & Exposure & \\
Pulp wall to cusp tip & $3.3(0.7)$ & $2.3(0.8)$ & $<0.0001^{*}$ \\
Pulp wall to lateral & $5.3(1.0)$ & $4.7(1.0)$ & $0.002^{*}$ \\
enamel distance & & & \\
\hline *: statistically significant difference at $\mathrm{P} \leq 0.05$ &
\end{tabular}

Table 3: Factors associated with occurrence of lateral exposure (logistic regression)

\begin{tabular}{|c|c|c|c|c|}
\hline \multirow[b]{2}{*}{ Variables } & \multicolumn{2}{|c|}{ Univariate regression } & \multicolumn{2}{|c|}{ Multivariate regression } \\
\hline & $p$ value & OR $(95 \%$ C.I.) & $p$ value & OR (95\% C.I.) \\
\hline Student gender: male vs female & $0.02^{*}$ & $2.86(1.16,7.02)$ & 0.48 & $3.14(0.13,27.64)$ \\
\hline Patient age & $<0.0001^{*}$ & $0.84(0.78,0.90)$ & 0.08 & $0.92(0.84,1.01)$ \\
\hline Patient gender: male vs female & 0.11 & $1.99(0.86,4.65)$ & 0.39 & $0.28(0.02,5.01)$ \\
\hline Arch: maxillary vs mandibular & 0.19 & $1.74(0.76,3.99)$ & $0.006^{*}$ & $10.34(1.93,15.29)$ \\
\hline Side: right vs left & 0.39 & $1.44(0.63,3.39)$ & 0.45 & $1.49(0.53,4.20)$ \\
\hline Teeth: anterior vs posterior & $<0.0001^{*}$ & $7.05(3.03,16.43)$ & $0.03^{*}$ & $3.53(1.13,11.02)$ \\
\hline Distance between pulp wall to cusp tip & $<0.0001^{*}$ & $0.10(0.05,0.21)$ & $<0.0001^{*}$ & $0.08(0.02,0.26)$ \\
\hline Distance between pulp wall to lateral enamel & $0.003^{*}$ & $0.53(0.35,0.81)$ & 0.63 & $0.87(0.48,1.56)$ \\
\hline
\end{tabular}

OR: odds ratio, C.I.: confidence interval, ${ }^{*}$ : statistically significant difference at $\mathrm{P} \leq 0.05$ 
tip were significantly associated with odds of exposure $(p=0.006,0.03$ and $<0.0001)$. Maxillary and anterior teeth (canines and incisors)were associated with higher odds of lateral exposure compared to mandibular and posterior $(\mathrm{OR}=10.34$ and 3.53). As the distance from the pulp wall to the cusp tip increased by $1 \mathrm{~mm}$, the odds of exposure decreased by about $90 \%(\mathrm{OR}=0.08)$.

\section{DISCUSSION}

The present paper evaluated the incidence of accidental pulpal exposure and its associated factors during the preparation of FPDs. Many studies have been conducted to study the complications involving the vital pulp after teeth preparation for FPD or full crown. ${ }^{6-9,11,12,19}$ However, an exhaustive search of the literature revealed few studies on pulpal exposure during tooth preparation for an FPD or single crown.

In the present study, we found $5.1 \%$ of lateral exposure and $0.2 \%$ occlusal exposure. This finding is in contrast to those Al-Khreisat ${ }^{20}$ and Gumus et al. ${ }^{21}$ who found at an exposure rate of $0.7 \%$ and $0.66 \%$, respectively. This difference in the findings could be because the majority of the patients in our study were young and in young patients the pulp chamber is considerably large. It has been advised that tooth preparations should be as minimal as possible to avoid pulp damage especially significant in young patients with large pulps. ${ }^{8}$ Also, in our study, the tooth preparation was done by undergraduate students and not dentists as in the Al-Khreisat ${ }^{20}$ research which could be the reason for higher pulp exposures. As occlusal exposure was infrequent, no further analysis was conducted for it.

We also measured the relationship of pulpal exposure with the distance from pulpal wall to cusp tips and pulpal wall to lateral enamel surface (Table 3) and found if the distance is more there was no exposure and vice versa. Also, the difference in the distance from the pulpal wall to enamel in cases where lateral exposure occurred and those where there was no exposure was statistically significant. As the distance from the pulp wall to the cusp tip increased by $1 \mathrm{~mm}$, the odds of pulpal exposure decreased by $90 \%$. Thus, it can be concluded that tooth anatomy plays an essential role in the occurrence of pulp exposure.

In the present study, the relationship of pulp exposure with patients age, gender, site (right /left), teeth (anterior/ posterior) and arch (maxillary/mandibular) were investigated. A multivariate analysis was performed for all independent variables, and the odds of pulpal exposure was significantly higher with the maxillary arch as compared to the mandibular $\operatorname{arch}(p$ value $=0.006)$ and with anterior teeth as compared to the posterior teeth $(p$ value $=0.003$ ). Similar findings were seen in a study conducted by Gumuset al. ${ }^{21}$ who reported canine teeth had the highest rate of pulpal exposure during tooth preparation. This could be attributed to the fact that the maxillary teeth require an indirect vision for tooth preparation and undergraduate students may not be comfortable using indirect vision ${ }^{9,11}$ Hence, it is easier for an inexperienced student to over-reduce these teeth during preparation for FPD.

The probable cause for more exposure in the anterior teeth and maxillary arch could be because the study was performed by the students and not by experienced dentists. Also, tooth preparation in maxillary teeth requires indirect vision, and the undergraduate students may not have enough experience with it.

Gumus et al. ${ }^{21}$ evaluated pulp exposures during tooth preparation in adult and pulpal exposure was found to increase significantly with the patient's age. The rate of pulpal exposure was lowest in the 21-30 age group and highest in the age group 70 and older. However, as the patients included in our study were between $20-40$ years of age (mean-35 years) in our study, no significant relation of pulp exposure and the age of the patient was observed.

\section{Limitations and Recommendations}

As the present study did not utilize an experimental design, causality cannot be established between the exposure of abutment teeth and the other clinical variables. Therefore, it is also difficult to determine which clinical factors are most effective in either causing or preventing pulpal exposures. Secondly, in the present study, only frank exposures were included. Further research evaluating clinical signs or symptoms of near exposure is recommended. Also, a comparison of dental students with experienced dentists may also be one of the directions for further research.

\section{CONCLUSION}

Within the limitations of the present study, it can be inferred that $5.1 \%$ of lateral and $0.2 \%$ occlusal exposures occurred in the present study.

The odd of pulpal exposure was significantly higher with the maxillary arch as compared to the mandibular arch and with anterior teeth as compared to the posterior teeth, hence, more caution should be exercised while preparing these teeth.

Also, knowledge of tooth anatomy is paramount in the success of tooth preparation.

\section{CLINICAL SIGNIFICANCE}

The present study draws the attention of the clinician towards the detrimental effect of tooth preparation on 
the vital pulp. The dentist must be extra careful while preparation of anterior and maxillary teeth for FPD to avoid pulpal exposure. The undergraduate students should undergo additional training for understanding the nuances of tooth preparation.

\section{REFERENCES}

1. Hebel K, Gajjar R, Hofstede T. Single-tooth replacement: bridge vs. implant-supported restoration. J Can Dent Assoc 2000;66(8):435-438.

2. Motta AB, Pereira LC, Da Cunha AR, Duda FP. All ceramic and porcelain fused to metal fixed partial dentures: a comparative study by 2D finite element analyses. J App Oral Sci 2007;15(5):399-405.

3. Hemmings K, Harrington Z. Replacement of missing teeth with fixed prostheses.Dental Update London 2004;31(3): 137-141.

4. Özcan M. Fracture reasons in ceramic fused to metal restorationn. J Oral Rehab 2003;30(3):265-269.

5. Anusavice KJ. Standardizing failure, success, and survival decisions in clinical studies of ceramic and metal-ceramic fixed dental prostheses. Dent Mater 2012;28(1):102-111.

6. Goodacre CJ, Bernal G, Rungcharassaeng K, Kan JY. Clinical complications in fixed prosthodontics. J Prosthet Dent 2003;90(1):31-41.

7. Cheung GSP, Lai SCN, Ng RPY. Fate of vital pulps beneath a metal-ceramic crown or a bridge retainer. Int End J 2005;38(8):521-30.

8. Christensen GJ. How to kill a tooth. J Am Dent Assoc 2005; 136(12): 1711.

9. Christensen GJ. Avoiding pulpal death during fixed prosthodontic procedures. J Am Dent Assoc 2002;133(11):1563-1564.

10. Borelli B, Sorrentino R, Goracci C, Zarone F, Ferrari M. In vitro analysis of residual tooth structure of maxillary anterior teeth after different prosthetic finish line preparations for full-coverage single crowns. J Oral Sci 2013;55(1):79-84.
11. Davis GR, Tayeb RA, Seymour KG, Cherukara GP. Quantification of residual dentine thickness following crown preparation. J Dent 2012;40(7):571-576.

12. Whitworth JM, Walls AWG, Wassell RW. Crowns and extracoronal restorations: endodontic considerations: the pulp, the root-treated tooth and the crown. Br Dent J 2002;192(6): 315-327.

13. Lee MM, Brian JR, Aura MT, Shah R, Musikant B, Deutsch A. Morphological measurements of anatomic landmarks in pulp chambers of human anterior teeth. J Endod 2007;33(2): 129-131.

14. Deutsch AS, Musikant BL, Gu S, Isidro M. Morphological measurements of anatomic landmarks in pulp chambers of human maxillary furcated bicuspids. J Endod 2005;31(8):570573.

15. Deutsch AS, Musikant BL. Morphological measurements of anatomic landmarks in human maxillary and mandibular molar pulp chambers. J Endod 2004;30(6):388-390.

16. Velmurugan N, Venkateshbabu N, Abarajithan M, Kandaswamy D. Evaluation of the pulp chamber size of human maxillary first molars: An institution based in vitro study. Ind J Dent Res 2008;19(2):92-94.

17. Ohashi Y. Research related to anterior abutment teeth of fixed partial denture. Shikagakuho. 1968;68:726.

18. Sherrard JF, Rossouw PE, Benson BW, Carrillo R, Buschang $\mathrm{PH}$. Accuracy and reliability of tooth and root lengths measured on cone-beam computed tomographs. Am J OrthodDentofacOrthop 2010;137(4):S100-S108.

19. Bidar M, Madani AS, Mohtasham N, Vardkar M, Jafarzadeh H. The Effect of Full Crown Preparation on Normal and Inflamed Pulp Tissue: An Animal Study. J Dent Mater Tech 2013;2(1):29-33.

20. AbeerS, Al-Khreisat AS. Early endodontic complications following fixed prosthodontic restorations. J Royal Med Serv 2010;17: 36-41.

21. Gumus HO, Kocaagaoglu HH, Aslan T, Albayrak H, Sagsen B. Prevalence of pulp exposures during tooth preparation for fixed prosthetics. Eur J Prosthod 2014;2(2):48-52. 